\title{
Band-gap shrinkage in $\boldsymbol{n}$-type-doped CdO probed by photoemission spectroscopy
}

\author{
Y. Dou, T. Fishlock, and R. G. Egdell* \\ Inorganic Chemistry Laboratory, South Parks Road, Oxford OX1 3QR, United Kingdom \\ D. S. L. Law and G. Beamson \\ Research Unit for Surfaces, Transforms and Interfaces, Daresbury Laboratory, Warrington, Cheshire, United Kingdom
}

(Received 27 January 1997)

\begin{abstract}
The influence of $n$-type doping $\mathrm{CdO}$ with $\mathrm{In}$ or $\mathrm{Y}$ has been investigated by high-resolution ultraviolet and $\mathrm{x}$-ray photoemission spectroscopy. It is found that core levels and valence band features suffer a shift to high binding energy due to doping. However this shift is less than the change in the width of the occupied conduction band. This provides a direct measurement of band gap shrinkage as a result of doping in an oxide semiconductor. [S0163-1829(97)50820-4]
\end{abstract}

There has been a long-standing interest in understanding the ways in which degenerate doping in semiconductors may influence the band gap of the host materials. ${ }^{1-3}$ Opticalabsorption experiments probe the energy separation between valence-band states and accessible conduction-band states, subject to the constraint of momentum conservation during the excitation process. The principal effect of doping is to block states at the bottom of the conduction band. In directgap materials with a conduction-band minimum and a valence-band maximum at the $\Gamma$ point the lowest-energy transitions originate from $\Gamma$ in undoped material but from valence-band states away from the zone center after doping. The so-called Moss-Burstein shift ${ }^{1}$ of the absorption onset is therefore influenced both by upward dispersion of the conduction band and (usually) the downward dispersion of the valence band with wave vector. This is illustrated in Fig. 1. However, analysis of these shifts is further complicated by the fact that electron-dopant interactions and electronelectron Coulomb and exchange interactions within the conduction band lead to a shrinkage or renormalization of the host band gap, reducing the magnitude of the shift in optical onset from $\Delta_{g}^{B M}$ to $\Delta_{g}^{B M}+\Delta_{g}^{N}$, where the band gap renormalization $\Delta_{g}^{N}$ is presumed to be negative. ${ }^{3}$

Post-transition-metal oxides such as $\mathrm{SnO}_{2}, \mathrm{In}_{2} \mathrm{O}_{3}, \mathrm{ZnO}$, and $\mathrm{Cd}_{2} \mathrm{SnO}_{4}$ are of particular interest because they are wide-gap materials which when $n$-type doped, combine the properties of high conductivity with transparency in the visible region. ${ }^{4}$ The visible window is limited by absorption due to interband excitation on the high-energy side and by high reflectivity below the conduction electron plasmon frequency at low energy. The technological importance of these materials has prompted a number of studies of Moss-Burstein shifts due to doping, including a full analysis of conductionand valence-band dispersion and band-gap renormalization in the case of $\mathrm{ZnO},{ }^{5} \mathrm{In}_{2} \mathrm{O}_{3},{ }^{6}$ and $\mathrm{SnO}_{2} \cdot{ }^{7}$ However, a limitation of the optical experiments is that they do not allow appraisal of individual roles of the three different contributions to the overall shift. In the present paper we demonstrate that high-resolution photoemission combined with electronenergy-loss spectroscopy provides an alternative approach to characterizing the effects of $n$-type doping in a posttransition metal oxide. A key feature of this approach is the direct observation of electrons in the conduction band of the host oxide, including the Fermi-Dirac onset. It is thus possible to obtain an accurate measurement of the position of the valence-band edge relative to the Fermi energy. Moreover, under optimal conditions the width of the occupied part of the conduction band can be observed: even when this is not possible, a good estimate of the width of the conduction band can be obtained from effective masses determined by electron-energy-loss spectroscopy (EELS). It is thus possible to make a direct determination of the renormalization of the band gap, as illustrated in Fig. 1. The present paper focuses on $\mathrm{CdO}$ which has a much narrower gap ${ }^{8}$ than the other oxides alluded to above and is not in itself widely used as a transparent, conducting window. Moreover, the band gap is indirect, ${ }^{9-11}$ which complicates the interpretation of optical spectra. However, $\mathrm{CdO}$ has a very low conduction electron effective mass, giving rise to relatively large shifts due to doping. As with the other oxides, Moss-Burstein shifts in optical absorption were first studied many years ago, although the importance of band-gap renormalization was not appreciated at this time. ${ }^{12,13}$

Polycrystalline ceramic samples of Y-and In-doped $\mathrm{CdO}$ were synthesized by firing a well-blended mixture of the stoichiometric quantities of $\mathrm{CdO}$ and $\mathrm{Y}_{2} \mathrm{O}_{3}$ or $\mathrm{In}_{2} \mathrm{O}_{3}$ at $880{ }^{\circ} \mathrm{C}$ for $2 \mathrm{~d}$ in the air in a recrystallized alumina boat. The product was slowly cooled to room temperature. For the sake of consistency, undoped $\mathrm{CdO}$ underwent the same calcination procedure. Up to doping levels of 3.5 at. $\% \mathrm{Y}$ and 2.3 at. \% In the samples showed a single cubic $\mathrm{CdO}$ rocksalt phase in $\mathrm{X}$-ray powder diffraction. Beyond these doping levels the powder patterns contained peaks due to $\mathrm{Y}_{2} \mathrm{O}_{3}$ or $\mathrm{CdIn}_{2} \mathrm{O}_{4}$. The samples were pressed into $10 \mathrm{~mm}$ diameter pellets at 5.5 $\mathrm{MPa}$, and sintered in air at $880^{\circ} \mathrm{C}$ for $8 \mathrm{~h}$. The occupancy of the conduction band was determined by a titrimetric method in which the $\mathrm{CdO}$ samples were dissolved in dilute sulfuric acid containing iodine: conduction electrons quantitatively reduce some of the iodine and the remainder is back titrated with sodium thiosulfate. ${ }^{14}$ The conduction-electron concentration estimated in this way was $0.85 \times 10^{20} \mathrm{~cm}^{-3}$ for nominally undoped $\mathrm{CdO}, 3.8 \times 10^{20} \mathrm{~cm}^{-3}$ for $3.5 \% \mathrm{Y}$-doped $\mathrm{CdO}$, and $4.8 \times 10^{20} \mathrm{~cm}^{-3}$ for $2.3 \%$ In-doped $\mathrm{CdO}$. These values are similar to those found in thin films where it is 


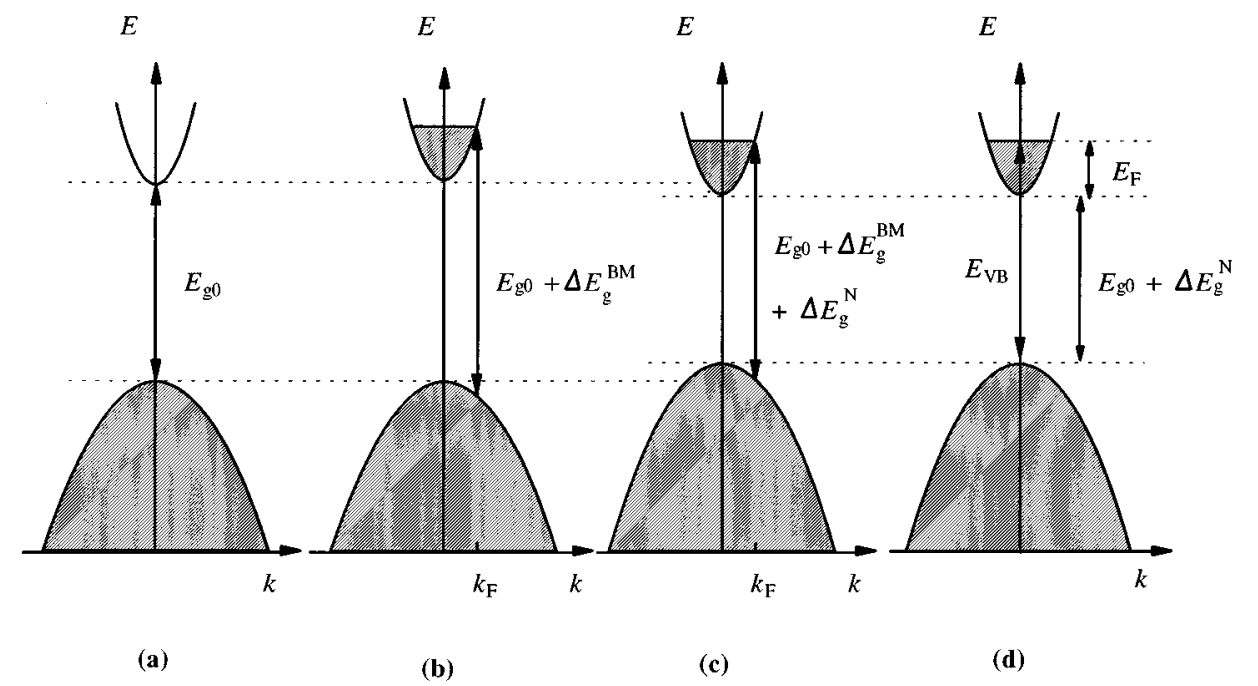

FIG. 1. Schematic energy-level diagrams showing effects of degenerate $n$-type doping in an oxide semiconductor. (a) Nominally undoped oxide with a direct optical band gap $\Delta_{g 0}$. (b) Doped oxide in which the optical gap has increased by $\Delta_{g}^{B M}$ due to blocking of states at the bottom of the conduction band. The shift depends on both conduction and valence-band dispersion. (c) Optical gap after band gap shrinkage. The new gap is seen to depend on both $\Delta_{g}^{B M}$ and $\Delta_{g}^{N}$ and the individual contributions to the new gap cannot be determined by optical absorption. (d) An illustration of the quantities which can be measured in the present experiment. These include the position of the valence-band-edge relative to the Fermi level $E_{V B}$ and the width of the occupied conduction-band $E_{f}$. The renormalized band gap $E_{g 0}$ $+\Delta_{g}^{N}$ can also be determined.

presumed that in the nominally undoped material the conduction electrons are associated with interstitial $\mathrm{Cd}$ donors.

Electron spectra were measured in two instruments. The first is an ESCALAB with facilities for excitation of photoemission spectra using unmonochromated $\mathrm{Al} K \alpha$ or $\operatorname{Mg} K \alpha$ Xrays or UV radiation from a noble-gas discharge lamp. Sample cleaning was achieved by annealing pellets for $2 \mathrm{~h}$ at around $400{ }^{\circ} \mathrm{C}$ (stub temperature) in the spectrometer preparation chamber (base pressure $<10^{-9} \mathrm{mbar}$ ). X-ray photoemission spectra (XPS) were found to be completely free of signals due to carbon or other contamination. The nominal analyzer resolution was set at $100 \mathrm{meV}$ for $\mathrm{He}(\mathrm{I})$ photoemission measurements. Procedures for stripping spectra of structure due to satellite radiation and for spectral calibration in this measurement have been described in detail elsewhere. ${ }^{15}$ Energy-loss spectra were measured in the same spectrometer using an incident electron beam derived from an electron gun usually used for low-energy electron diffraction (LEED) studies, again as described elsewhere. ${ }^{15}$ The second instrument is a Scienta ESCA 300 XPS spectrometer incorporating a rotating anode $\mathrm{x}$-ray source with seven-crystal $\mathrm{x}$-ray monochromator, $300 \mathrm{~mm}$ mean radius electron-energy analyzer, and parallel electron-detection system. The overall effective resolution in this instrument was $350 \mathrm{meV}$. The instrument is regularly calibrated using the Fermi level and core levels from an ion-bombarded silver foil. The cleaning procedures in the Scienta spectrometer were similar to those in the ESCALAB, except that heating was via a resistive P8 probe heater.

Figure 2 shows UV photoemission spectra of nominally undoped $\mathrm{CdO}$ and of maximally $\mathrm{Y}$ - and In-doped $\mathrm{CdO}$ in the binding energy range up to $4 \mathrm{eV}$. This region encompasses the conduction-band, bulk band gap, and valence-band edge. Straddling zero-binding-energy is a Fermi-Dirac-like cutoff associated with electrons in the $\mathrm{Cd} 5 s$ conduction band. The

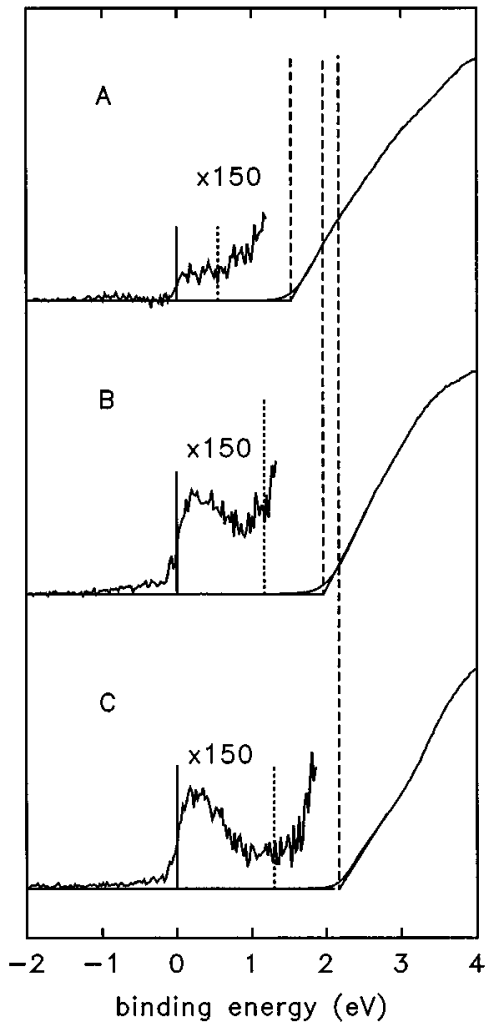

FIG. 2. He(I) photoemission spectra of (a) nominally-undoped $\mathrm{CdO}$, (b) $3.5 \%$ Y-doped $\mathrm{CdO}$, and (c) 2.3\% In-doped CdO. Structure due to satellite radiation has been stripped from the spectra. Binding energies are relative to the Fermi energy, which is indicated by bold vertical lines. The positions of the $\mathrm{O} 2 p$ valence-band edges determined by a linear extrapolation procedure are indicated with bold dashed lines. The positions of the bottoms of the conduction bands estimated from measured surface-plasma energies through application of Eq. (5) are indicated by light dashed lines. 
conduction-band feature is much weaker and narrower in the nominally undoped $\mathrm{CdO}$ than for the $\mathrm{Y}$ - or In-doped samples, in accordance with the lower-carrier concentration. Moreover when binding energies are referred to the Fermi level it is clear that the valence-band edge shows the expected shift to high-binding energy due to the filling of the conduction band in the doped samples. This conclusion is confirmed by high-resolution XPS. Here it is found that both the valence-band edge and peaks in the valence band show shifts to high binding energy with doping, as illustrated in Fig. 3, where the shift of a sharp valence-band peak at about $6 \mathrm{eV}$ binding energy is highlighted. Moreover the $4 d$ corelevel peaks also show pronounced shifts. A complete set of binding energies is given in Table I, where it is seen that within the experimental uncertainty of about $0.05 \mathrm{eV}$ all three features alluded to above show the same shift.

In order to understand the magnitude of the shifts, it is necessary to consider the widths of the occupied part of the conduction band. In a simple free-electron model the occupied bandwidth is given by $E_{f}$ where,

$$
E_{f}=\left(\frac{h}{2 \pi}\right)^{2} \frac{\left(3 \pi^{2} N\right)^{2 / 3}}{2 m^{*}} .
$$

Here $m^{*}$ is the electron effective mass and $N$ is the carrier concentration.

However, it is well known for narrow-gap oxides such as $\mathrm{CdO}$ that the effective mass varies strongly as a function of carrier concentration. We have, therefore, used EELS to probe effective-mass variations. Typical energy-loss spectra excited at $200 \mathrm{eV}$ beam energy are shown in Fig. 4. At low energy, a well-defined peak is observed with the intensity of the order expected ${ }^{16}$ for pure surface loss (as opposed to bulk loss). The surface-loss frequency is given by

$$
\omega_{s p}^{2}=\frac{N e^{2}}{\varepsilon_{0}[\varepsilon(\infty)+1]},
$$

where $e$ is the electronic charge, $\varepsilon_{0}$ is the permittivity of free space, and $\varepsilon(\infty)$ is the high-frequency dielectric constant which is known to be 5.0. The plasmon is built up from electron-hole excitations centered around the Fermi energy ${ }^{17}$ and the effective mass relevant to EELS therefore depends on the dispersion of energy with wave vector at $E_{f}$,

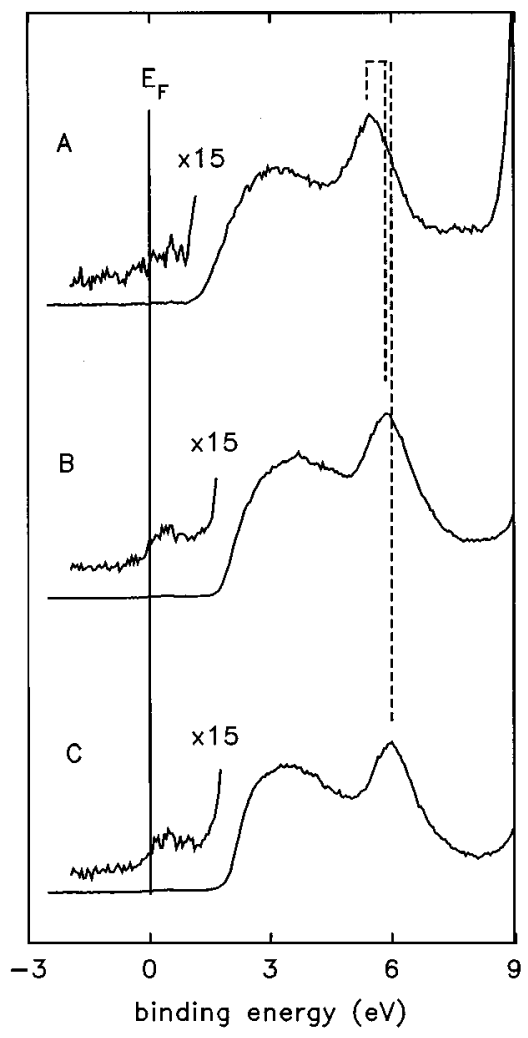

FIG. 3. High-resolution valence-band XPS excited with monochromatized Al $K \alpha$ x-rays for (a) nominally-undoped $\mathrm{CdO}$, (b) $3.5 \%$ Y-doped $\mathrm{CdO}$, and (c) $2.3 \%$ In-doped $\mathrm{CdO}$. Binding energies are given relative to the Fermi energy which is indicated by a solid line. Note the shift in the sharp valence-band peak at about $6 \mathrm{eV}$ binding energy.

$$
\left|\frac{d E}{d k}\right|_{E=E_{f}}=\left(\frac{h}{2 \pi}\right)^{2} \frac{k_{f}}{m^{*}} .
$$

We have found from an extensive series of measurements in EELS of surface-plasmon energies at different doping levels that the effective mass shows an essentially linear variation with carrier concentration $N$,

$$
m^{*}=m_{0}^{*}+c N
$$

\begin{tabular}{|c|c|c|c|}
\hline & $\mathrm{CdO}$ & $3.5 \%$ Y-doped $\mathrm{CdO}$ & 2.3\% In-doped CdO \\
\hline Valence-band onset & $1.53 \pm 0.05$ & $1.96 \pm 0.05(0.43)$ & $2.17 \pm 0.05(0.64)$ \\
\hline $\begin{array}{l}\text { Valence-band peak } \\
\mathrm{BE}^{\mathrm{c}}\end{array}$ & $5.40 \pm 0.05$ & $5.90 \pm 0.05(0.50)$ & $6.00 \pm 0.05(0.60)$ \\
\hline $\mathrm{Cd} 4 d$ core-level $\mathrm{BE}$ & $10.60 \pm 0.05$ & $11.05 \pm 0.05(0.45)$ & $11.25 \pm 0.05(0.65)$ \\
\hline $\begin{array}{l}\text { Conduction-band } \\
\text { width estimated from } \\
\text { EELS data }^{d}\end{array}$ & 0.54 & $1.19(0.65)$ & $1.32(0.78)$ \\
\hline
\end{tabular}

TABLE I. Binding energies and bandwidths in $n$-type-doped CdO.$^{\text {a,b }}$

${ }^{\mathrm{a}}$ All values are in $\mathrm{eV}$.

${ }^{\mathrm{b}}$ Figure in parentheses give shifts relative to $\mathrm{CdO}$.

${ }^{\mathrm{c}}$ Valence-band peak highlighted in Fig. 2.

${ }^{\mathrm{d}}$ The effective mass is assumed to vary within the conduction band in accordance with Eq. (4). 


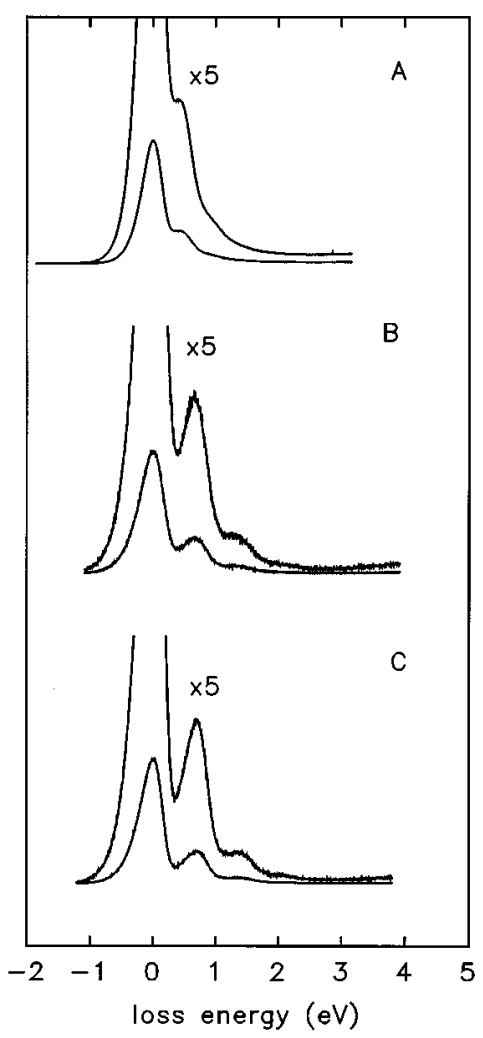

FIG. 4. Energy-loss spectra of (a) nominally-undoped CdO, (b) $3.5 \%$ Y-doped $\mathrm{CdO}$, and (c) $2.3 \%$ In-doped $\mathrm{CdO}$ excited with 200 $\mathrm{eV}$ electron beam showing loss peaks due to excitation of conduction-electron surface plasmons.

where $c$ is a constant. Experimentally we find $m_{0}^{*} / m_{0}$ $=0.121$ and $c / m_{0}=0.0315 \times 10^{-20} \mathrm{~cm}^{3}$, where $m_{0}$ is the electron rest mass. This linear variation is in agreement with previous work. ${ }^{13}$ The Fermi energy now depends on the integral

$$
E_{f}=\int_{0}^{N} \frac{h^{2}}{4\left(3 \pi^{2} n\right)^{1 / 3}\left(m_{0}^{*}+c n\right)} d n .
$$

The calculated conduction-band widths obtained in this way are given in Table I. The variation of $m^{*}$ within the band gives rise to much broader conduction bands than would be expected if the mass at the Fermi level applied throughout the band. The calculated bandwidths are also indicated on Fig. 2. The agreement with experiment is satisfactory, subject to the reservation that it is difficult to define the position of the bottom of the conduction band in nominally undoped $\mathrm{CdO}$ due to its very weak intensity and the occurrence of states in the bulk band gap just above the valence-band edge. These probably arise from $\mathrm{Cd}$ atoms in surface sites where $5 s-5 p$ mixing induced by the electric-field gradient at the surface pushes $\mathrm{Cd} 5 s$ states down into the band gap to give a doubly occupied $s p$ hybrid state: similar states exist on $\mathrm{SnO}_{2}$ surfaces. ${ }^{18}$ Despite this difficulty, it is clear that the binding-energy shifts of the valence-band edges in In- and $\mathrm{Y}$-doped $\mathrm{CdO}$ are significantly less than expected from the increased width of the conduction band. The discrepancies correspond to the shrinkage of the band gap, which is thus estimated to be $0.22 \pm 0.05 \mathrm{eV}$ for $3.5 \% \mathrm{Y}$-doped $\mathrm{CdO}$ and $0.14 \pm 0.15 \mathrm{eV}$ for $2.3 \% \mathrm{In}$-doped $\mathrm{CdO}$. These estimates are of the order of magnitude expected from calculations ${ }^{6}$ on Sn-doped $\mathrm{In}_{2} \mathrm{O}_{3}$ using Heine-Abarenkov pseudopotentials to deal with the electron impurity interaction and the randomphase approximation to account for electron-electron interactions. In this work it was found that at a carrier concentration of $4 \times 10^{20} \mathrm{~cm}^{-3}$ the contributions to the band gap narrowing from electron-impurity and electron-electron interactions were about 0.05 and $0.2 \mathrm{eV}$, respectively.

In summary, we have developed a method for measuring the band gap shrinkage in degenerately doped oxide semiconductors and have applied this technique to $\mathrm{CdO}$. It should be straightforward to extend this approach to other oxides such as $\mathrm{SnO}_{2}$ and $\mathrm{In}_{2} \mathrm{O}_{3}$.

*Author to whom correspondence should be sent. Electronic address: Egdell@ermine.ox.ac.uk

${ }^{1}$ E. Burstein, Phys. Rev. 93, 632 (1954).

${ }^{2}$ R. A. Abram, G. J. Ress, and B. L. H. Wilson, Adv. Phys. 27, 799 (1978)

${ }^{3}$ K. F. Berggren and B. E. Sernelius, Phys. Rev. B 24, 1971 (1981).

${ }^{4}$ H. Köstlin, Festkoerperprobleme 22, 229 (1982).

${ }^{5}$ Z. C. Jin, I. Hamberg, C. G. Granquist, B. E. Sernelius, and K. F. Berggren, Thin Solid Films 164, 381 (1988).

${ }^{6}$ I. Hamberg, K. F. Berggren, L. Engstrom, C. G. Granquist, and B. E. Sernelius, Phys. Rev. B 30, 3240 (1984).

${ }^{7}$ G. Sanon, R. Rup, and A. Mansingh, Phys. Rev. B 44, 5672 (1991).

${ }^{8}$ H. Finkenrath, in Physics of II-VI and I-VII Compounds, Semimagnetic, Semiconductors, edited by O. Madelung, M. Schulz, and H. Weiss, Landolt-Börnstein, New Series, Group III, Vol.

17 (Springer-Verlag, Berlin, 1982), p. 162 and references therein.

${ }^{9}$ H. Köhler, Solid State Commun. 11, 1687 (1972).

${ }^{10}$ J. C. Boettger and A. B. Kunz, Phys. Rev. B 27, 1359 (1982).

${ }^{11}$ J. E. Jaffe, R. Pandey, and A. B. Kunz, Phys. Rev. B 43, 14030 (1991).

${ }^{12}$ H. Finkenrath, Z. Phys. 159, 112 (1960).

${ }^{13}$ H. Finkenrath and M. von Ortenberg, Z. Angew. Phys. 23, 323 (1967).

${ }^{14}$ M. P. Shatlock and G. E. Machiel, J. Chem. Phys. 81, 859 (1984).

${ }^{15}$ Y. Dou and R. G. Egdell, Phys. Rev. B 53, 15405 (1996).

${ }^{16}$ H. Ibach and D. L. Mills, Electron Energy Loss Spectroscopy and Surface Vibrations (Academic, New York, 1982).

${ }^{17}$ T. Inaoka, D. M. Newns, and R. G. Egdell, Surf. Sci. 186, 290 (1987).

${ }^{18}$ I. Manassidis, J. Goniakowski, L. N. Kantorovitch, and M. J. Gillan, Surf. Sci. 339, 258 (1995). 